OPEN ACCESS

Edited by:

Junji Xing,

Houston Methodist Research Institute,

United States

Reviewed by:

Kevin Beier,

University of California,

Irvine, United States

Vikas Duhan,

The University of Queensland,

Australia

*Correspondence:

Gencheng Han

genchenghan@163.com

Renxi Wang

wang_renxi@hotmail.com

Specialty section:

This article was submitted to

Molecular Innate Immunity,

a section of the journal

Frontiers in Immunology

Received: 13 February 2021

Accepted: 23 April 2021

Published: 07 May 2021

Citation:

Li G, Tang L, Hou C, Wang Z, Gao Y,

Dou S, Mo R, Hao Y, Gao Z, Li Y,

Dong J, Zhang J, Shen $B$, Wang $R$ and Han G (2021) Peripheral Injection of

Tim-3 Antibody Attenuates VSV

Encephalitis by Enhancing

MHC-I Presentation.

Front. Immunol. 12:667478.

doi: 10.3389/fimmu.2021.667478

\section{Peripheral Injection of Tim-3 Antibody Attenuates VSV Encephalitis by Enhancing MHC-I Presentation}

\author{
Ge Li ${ }^{1}$, Lili Tang ${ }^{1}$, Chunmei Hou ${ }^{1}$, Zhiding Wang ${ }^{1}$, Yang Gao ${ }^{1}$, Shuaijie Dou ${ }^{1}$, \\ Rongliang Mo ${ }^{1}$, Ying $\mathrm{Hao}^{1}$, Zhenfang Gao ${ }^{1}$, Yuxiang $\mathrm{Li}^{1}$, Jie Dong ${ }^{1}$, Jiyan Zhang ${ }^{1}$, \\ Beifen Shen ${ }^{1}$, Renxi Wang ${ }^{2 *}$ and Gencheng Han ${ }^{1 *}$ \begin{abstract}
2 Beijing Institute of Brain Disorders, Laboratory of Brain Disorders, Ministry of Science and Technology,
\end{abstract} \\ 1 Department of Neuroimmune and Antibody Engineering, Beijing Institute of Basic Medical Sciences, Beijing, China, \\ Collaborative Innovation Center for Brain Disorders, Capital Medical University, Beijing, China
}

Viral encephalitis is the most common cause of encephalitis. It is responsible for high morbidity rates, permanent neurological sequelae, and even high mortality rates. The host immune response plays a critical role in preventing or clearing invading pathogens, especially when effective antiviral treatment is lacking. However, due to blockade of the blood-brain barrier, it remains unclear how peripheral immune cells contribute to the fight against intracerebral viruses. Here, we report that peripheral injection of an antibody against human Tim-3, an immune checkpoint inhibitor widely expressed on immune cells, markedly attenuated vesicular stomatitis virus (VSV) encephalitis, marked by decreased mortality and improved neuroethology in mice. Peripheral injection of Tim-3 antibody enhanced the recruitment of immune cells to the brain, increased the expression of major histocompatibility complex-I (MHC-I) on macrophages, and as a result, promoted the activation of VSV-specific CD8 ${ }^{+} \mathrm{T}$ cells. Depletion of macrophages abolished the peripheral injection-mediated protection against VSV encephalitis. Notably, for the first time, we found a novel post-translational modification of MHC-I by Tim-3, wherein, by enhancing the expression of MARCH9, Tim-3 promoted the proteasome-dependent degradation of $\mathrm{MHC}$-I via K48-linked ubiquitination in macrophages. These results provide insights into the immune response against intracranial infections; thus, manipulating the peripheral immune cells with Tim-3 antibody to fight viruses in the brain may have potential applications for combating viral encephalitis.

\footnotetext{
Keywords: encephalitis, vesicular stomatitis virus, Tim-3, macrophages, major histocompatibility complex-I, ubiquitination
}

Abbreviations: Tim-3, T- cell immunoglobulin and mucin domain-3; VSV, vesicular stomatitis virus; MHC-I, Major Histocompatibility Complex calss-1; pMDM, peripheral monocyte derived macrophages. 


\section{INTRODUCTION}

Viral encephalitis is defined as a pathological inflammation of the brain parenchyma secondary to viral infection. Worldwide, rabies and Japanese encephalitis viruses are responsible for an estimated annual mortality of 60,000 and 17,000 people, respectively $(1,2)$. Encephalitis is associated with appreciable mortality and high rates of permanent neurological impairment in survivors, and in most cases, there is no available antiviral therapy. Therefore, insight into its pathogenesis is urgently needed (3). Encephalitis may result from direct viral exposure, hematogenous spread, or retrograde infection of the nervous system. Meanwhile, limited vaccine availability and efficiency challenge the prevention of viral encephalitis (4). Innate immune cells, including resident microglia, recruited peripheral monocyte-derived macrophages (pMDM), and recruited peripheral $\mathrm{T}$ cells, play critical roles in viral encephalitis prevention or clearance (5). However, the mechanisms by which resident cells cooperate with the peripheral immune cells to fight viral encephalitis are still undetermined.

Central nervous system (CNS) is an immune-privileged organ towing to the presence of the blood-brain barrier (BBB) and relatively low number of surveilling peripheral immune cells within the brain parenchyma (6). However, during neuroinfection or chronic neuroinflammation, peripheral immune cells infiltrate the CNS and accumulate near sites of infection (7). Microglia are the CNS-resident mononuclear phagocytic cells that are typified by a unique ramified shape and distinctive gene expression (8). Unlike blood-derived macrophages, most microglia are derived from a yolk sac progenitor that seed the brain during early development ( 9 , 10). Recent studies suggest that microglia are important for neurogenesis, synapse maintenance, neuroimmune homeostasis, and neuronal function, thereby indicating that these cells maintain a healthy brain by performing a multitude of functions (11-15). During viral encephalitis, the absence of microglia leads to more rapid viral replication, which allow the virus to evade the immune response. Microglia are reportedly required between days 0 and 6 post-infection with depletion at later stages having no effect on survival, thereby suggesting that microglia play a role predominantly in the early stages of infection. As a consequence of cytokine and chemokine secretion, viral infection of the CNS results in recruitment of innate and adaptive immune cells to the brain. Virus-specific $\mathrm{CD}^{+} \mathrm{T}$ cells, which are detectable within the brain 5-7 days post-infection, are critical for viral clearance $(16,17)$. Spontaneous recruitment of peripheral immune cells seems to be insufficient under neuroinflammation or neuroinfection (18); thus, it is of great interest to identify mechanisms of recruitment and methods of manipulating this process.

In recent years, immune checkpoint inhibitors, such as programmed cell death protein-1 (PD-1), cytotoxic Tlymphocyte-associated protein 4 (CTLA-4), and T cell immunoglobulin and mucin domain-containing protein 3 (Tim-3), have attracted considerable attention as these molecules play critical roles in maintaining immune homeostasis, and their dysregulation is associated with many immune-related diseases, tumor development, chronic infections, and autoimmune diseases. Antagonists against PD1 , which can systematically enhance the immune response, are effective in many tumors. Interestingly, a report showed that enhanced systemic immune response induced by a PD-1 antagonist may also have a therapeutic role in Alzheimer's disease $(19,20)$. Although the underlying mechanisms remain to be determined, the data suggest that immune checkpoint molecules may be widely targeted in many immunerelated disorders.

Tim- 3 was initially identified on activated Th1, Th17, and Tc1 cells, and engagement of Tim-3 reportedly leads to $\mathrm{T}$ cell tolerance or failure (21). It was later observed that Tim-3 is also expressed on innate immune cells such as macrophages (22). Tim-3 plays a critical role in maintaining the tolerance of macrophages in both tumors and infectious diseases $(23,24)$. Compared with PD-1, which is mainly expressed on T cells, the wider distribution of Tim-3 implies that it may play crucial roles in modulating the systemic immune response. Thus, it is of great interest whether peripheral injection of Tim-3 antagonist may enhance immune response systematically, and whether it can be helpful in fighting against neuroinfection.

Here, we employed a vesicular stomatitis virus (VSV) encephalitis mouse model to investigate the mechanism by which innate and adaptive immune cells thwart neuroinvasion with or without modulation of the peripheral immune response by Tim-3 antagonist. Our data showed that peripheral injection of Tim-3 antibody significantly enhanced the migration and antigen-presenting activity of macrophages, which contributed to virus control in viral encephalitis.

\section{MATERIALS AND METHODS}

\section{Mice}

Male C57BL/6 mice (6-8 weeks old) were purchased from Beijing Vital River Laboratory Animal Technology Co. Ltd., China. All mice were bred and maintained under specific pathogen-free conditions, and the experimental protocol was approved by the Ethics Committee of Animal Experiments of the Institute of Military Cognitive and Brain Sciences (permit number: AMMS2020-0166). All efforts were made to minimize animal suffering.

\section{Cell Culture and Transfection}

RAW264.7, Vero, and HEK293T cells (American Type Cell Culture, Manassas, VA, USA) were maintained in Dulbecco's Modified Eagle's Medium (DMEM; Gibco, USA) supplemented with $10 \%$ fetal bovine serum (FBS; Gibco, USA). Mouse peritoneal macrophages were collected as previously described (24). For transient expression, the vector (pcDNA3.1) alone, plasmids coding full-length human Tim-3 (Tim-3-pcDNA3.1), or plasmids coding membrane associated ring- $\mathrm{CH}$-type finger-9 (MARCH9) were transfected into HEK293T cells for $48 \mathrm{~h}$. 


\section{In Vivo Experimental VSV Infection}

VSV was a gift from Prof. Minghong Jiang at the Institute of Basic Medicine, Chinese Academy of Medical Sciences. VSV was cultivated as previously described (25). Mice were anesthetized by intraperitoneal injection of pentobarbital $(150 \mathrm{mg} / \mathrm{kg})$. Intracranial injections were performed on the left side, $1.5 \mathrm{~mm}$ lateral and $2.0 \mathrm{~mm}$ rostral of the bregma at $2.0 \mathrm{~mm}$ depth using micro-syringes from Gaoge (Shanghai, China) controlled by a stereotactic injector from Longer (Shanghai, China). Next, $2 \mu \mathrm{L}$ VSV was injected at a concentration of $1 \times 10^{6} \mathrm{pfu} / \mathrm{g}$ for $10 \mathrm{~min}$ and the needle was kept in place for an additional $10 \mathrm{~min}$ before removal.

The monoclonal antibody against human Tim-3 (clone A3) was originally obtained by screening human natural phage antibodies library using recombinant human Tim-3 protein as bait. To test the efficacy of anti-Tim-3 antibody in VSV infection, mice were injected intraperitoneally with $10 \mathrm{mg} / \mathrm{kg}$ of neutralizing antibody specific for Tim-3 or human IgG1 isotype control antibody (BioLegend, USA) diluted in $200 \mu \mathrm{L}$ of phosphate-buffered saline (PBS) both $48 \mathrm{~h}$ and $24 \mathrm{~h}$ before and after injection with VSV.

\section{Quantification of Viral Load}

VSV load in brain tissue samples was determined by TCID50 assay (50\% tissue culture infectious dose), which is a method to measure the amount of infectious virus in a sample by determining the highest dilution of the sample that can infect $50 \%$ of the cells in a culture. Virus mRNA replication was analyzed by reverse transcriptase quantitative-polymerase chain reaction (RT-PCR (26);. Brain tissues were collected on day 5 after infection, transferred to lysing matrix tubes, and incubated in $1000 \mu \mathrm{L}$ DMEM (10\% FBS). Serial 10-fold dilutions of supernatant were added to Vero cell monolayers in 96-well plates and they were incubated for $72 \mathrm{~h}$ at $37^{\circ} \mathrm{C}$. Endpoints of cytopathic effect were observed, and TCID50 was determined using the ReedMuench method. For RT-PCR, samples were subjected to RNA extraction and cDNA synthesis, as described previously. Then, cDNA was amplified using SYBR Green I Master Mix (Roche, Basel, Switzerland) and a LightCycler 480 PCR system (Roche) with primers targeting the VSV gene (forward primer: 5'-CAAGTCAAAATGCCCAAGAGTCACA-3' and reverse primer: 5'-TTTCCTTGCATTGTTCTACAGATGG-3'). Results are expressed as the relative number of genome copies of VSV per sample.

\section{Behavioral Assessment}

Behavioral changes in mice following VSV infection were recorded using the open-field test (OFT) and automated computer-assisted method (CatWalk, Noldus Information Technology Inc., Netherlands). The OFT was used to examine both locomotor activity and anxious behavior. The floor of the open field was divided into 16 equal rectangles using black lines, wherein set area was zonel and the rest of the rectangles were zone2. Fusion software (ANY-maze) analyzed various parameters based on recorded activity, including total distance, time in zone1, and average duration of visit to zone1. Each mouse was individually placed in the middle of the apparatus and allowed to explore for $2 \mathrm{~min}$. Animals were tested twice on consecutive days on the OFT to examine habituation.

Gait analysis was performed on mice that could walk using the CatWalk system. Five trials per mouse, with a maximum of $10 \mathrm{~s}$ to traverse the glass plate, were performed. The gait analysis system is designed to dynamically measure the footprints of moving animals and assess any locomotor deficits in animal models; these are widely used in viral encephalitis studies. The regularity index (\%) is a fractional measure of inter-paw coordination, which expresses the number of normal step sequence patterns relative to the total number of paw placements. In healthy, fully coordinated animals, the regularity index is close to $100 \%$. Swing speed provides the speed of the paws during the swing phase. Meanwhile, stride length characterizes the distance between the placement of the paw and subsequent placement of the same paw. These data were analyzed using the Catwalk software.

\section{Flow Cytometry}

Brain tissues were excised from mice and individually homogenized. Then, the tissues were incubated with $1 \mathrm{mg} / \mathrm{mL}$ collagenase D (Roche) and $15 \mu \mathrm{g} / \mathrm{mL}$ DNase (Invitrogen, USA) for $40 \mathrm{~min}$ at $37^{\circ} \mathrm{C}$, filtered through a $70 \mu \mathrm{M}$ filter, suspended in $40 \%$ Percoll (GE Healthcare, USA), in RPMI 1640, overlayered with $70 \%$ Percoll, and centrifuged for $20 \mathrm{~min}$ at $4^{\circ} \mathrm{C}$ and 2000 $\mathrm{rpm}$. Cells at the $40 \%-70 \%$ interface were collected, diluted in PBS, centrifuged for $8 \mathrm{~min}$ at $1500 \mathrm{rpm}$, and resuspended as a single-cell suspension for analysis. Spleens were dissected and scrubbed through a $70 \mu \mathrm{M}$ nylon mesh cell strainer, then subjected to density gradient centrifugation (Ficoll-Hypaque, TBD Science, Tianjin, China) for $20 \mathrm{~min}$ at $24^{\circ} \mathrm{C}$ and 2000 rpm. The cells at the Ficoll interface were collected, diluted in PBS, and centrifuged for $8 \mathrm{~min}$ at $1500 \mathrm{rpm}$. The cells were then resuspended in flow cytometry wash buffer (2\% FBS in PBS) for staining according to standard protocols using the following antibodies: CD11b-Percp, CD45-FITC, CD4-APC, and CD8BV510 (Biolegend, San Diego, CA, USA). The gating strategy employed to quantify frequencies of infiltrating and resident immune cells is as described previously (5). Total immune cells were initially gated based on expression of the leukocyte common antigen CD45 and side scatter, and further gated by $\mathrm{CD} 11 \mathrm{~b}$ expression to distinguish microglia $\left(\mathrm{CD} 11 \mathrm{~b}^{+} \mathrm{CD} 45^{\mathrm{Low}}\right)$ (P1) from non-myeloid infiltrating lymphocytes (CD11b CD45 ${ }^{\text {hi }}$ (P3) and infiltrating monocytes derived macrophages $\left(\mathrm{CD} 11 \mathrm{~b}^{+} \mathrm{CD} 45^{\mathrm{hi}}\right)(\mathrm{P} 2)(27)$. Tetramer staining of brain and spleen cells was performed using a PE-conjugated MHC-I $\left(\mathrm{H} 2 \mathrm{~K}^{\mathrm{b}}\right)$ tetramer folded with the VSV epitope peptide RGYVYQGL (MBL, Japan (28);. Data were acquired on a FACSCalibur flow cytometer (BD Biosciences, USA) and analyzed using Flow Jo software.

\section{Western Blot Analysis and Co-immunoprecipitation}

For the western blot analysis, cells were harvested and lysed in lysis buffer supplemented with protease and phosphatase 
inhibitors (Sigma Aldrich). Samples were separated by $10 \%$ sodium dodecyl sulfate-polyacrylamide gel electrophoresis (SDS-PAGE), and the expression of the indicated proteins with the indicated antibodies was examined.

For co-immunoprecipitation, cells were collected $24 \mathrm{~h}$ after transfection and lysed in lysis buffer supplemented with a protease inhibitor cocktail. After centrifugation for $15 \mathrm{~min}$ at $12000 \mathrm{rpm}$ and $4^{\circ} \mathrm{C}$, the supernatant was collected and incubated with Protein A/G Sepharose beads (SC-2003, Santa Cruz) coupled to specific antibodies overnight with slow rotation at $4^{\circ} \mathrm{C}$. The next day, beads were washed three times with highsalt wash buffer and three times with low-salt wash buffer, and finally eluted by boiling for $10 \mathrm{~min}$ with $5 \times$ sample buffer (as indicated). Precipitates were fractionated using SDS-PAGE at appropriate concentrations (as indicated).

\section{Ubiquitination Assay}

To analyze the ubiquitination of the major histocompatibility complex-I (MHC-I) in HEK293T cells, plasmids encoding ubiquitin with HA tag (HA-ubiquitin), or Ubiquitin K48 with HA tag (Ub-k48) which mediates proteasomal-dependent degradation of the target protein, were transfected into HEK293T cells for $24 \mathrm{~h}$ and then treated with $20 \mu \mathrm{M}$ MG132, a proteosome inhibitor, for $6 \mathrm{~h}$ before harvesting. Cells were lysed with immunoprecipitation lysis buffer (1\% NP-40, $20 \mathrm{mM}$; Tris- $\mathrm{HCl}$, $150 \mathrm{mM}$; NaCl, $5 \mathrm{mM}$; EDTA, $1 \mathrm{mM} ; \mathrm{Na}_{3} \mathrm{VO}_{4}, 0.5 \%$; and sodium deoxycholic acid and complete protease inhibitor cocktail [Roche], $\mathrm{pH}$ 7.5), and then whole-cell lysates were immunoprecipitated with antibodies against Flag-tag (F1804), followed by analysis of ubiquitination of MHC-I with MHC-I antibody (Proteintech,15240-1-AP). Precipitates were fractionated using SDS-PAGE at appropriate concentrations (as indicated).

\section{Real-Time Quantitative RT-PCR}

Total RNA was extracted using TRIzol reagent by following the manufacturer's instructions. Real-time quantitative RT-PCR analysis was performed using SYBR Green I Master Mix (Roche, Basel, Switzerland) and a Light Cycler 480 PCR system (Roche). The relative expression of the gene of interest was determined using the $2^{-\Delta \Delta \mathrm{Ct}}$ method, with $18 \mathrm{~S}$ ribosomal mRNA (18S) used as the internal control. Primers used for RT-PCR are listed in Table S1.

\section{Histopathology and Immunofluorescence}

Brains were post-fixed overnight with $4 \%$ formaldehyde after their removal from the mice. Sections were made in a rostral to caudal fashion, and were mounted and stained with hematoxylin and eosin. Sections were analyzed for brain pathology and signs of inflammation, focusing on the regions of the cortex/meninges. For immunofluorescence, anti-MHC-I-VSV-tetramers and antiCD8 antibodies were used (MBL,TS-M529-1). Images were obtained at 200-400× magnification using an Olympus BX51 optical microscope equipped with a camera.

\section{Statistical Analysis}

Statistical significance was determined using a one-way ANOVA followed by a two-tailed Student's $t$-test. For the mouse survival study, Kaplan-Meier survival curves were generated and analyzed for significance using GraphPad Prism 8.0.A P value $<0.05$ was considered statistically significant.

\section{RESULTS}

\section{Peripheral Injection of Tim-3 Antibody Attenuates VSV Encephalitis in Mice}

To determine whether manipulating the peripheral immune response affects the outcome of VSV encephalitis, a monoclonal antibody against human Tim-3 (clone A3) was injected into mice on days $-2,0$, and 2 of intracerebral VSV injection. This antibody of Tim-3 binds to both human and mouse Tim-3 (Figures S1A, B) and showed excellent neutralizing activity in human and mouse macrophage cell lines in vitro (Figures S1C, D) and in healthy mice in vivo (Figure S2). Peripheral injection of Tim-3 antibody increased the survival of mice with VSV encephalitis than the isotype control mice (Figure 1A). We then examined the viral load, viral replication, and tissue damage of the brain tissues acquired from mice with VSV encephalitis, with or without Tim-3 antibody injection. Figures 1B, C show that peripheral injection of Tim-3 antibody inhibited viral replication and attenuated tissue damage. Figure 1D shows that Tim-3 antibody injection markedly decreased meningeal injury and perivascular cuffing. Finally, we examined the effects of Tim-3 antibody injection on the neuroethology of mice using open-field test (OFT) and CatWalk assay. The data showed that mice that received Tim-3 antibody moved a longer distance in the open field (Figure 1E) and showed a higher regularity index (Figure 1F) than mice that received the control antibody. Spontaneous locomotor activity and locomotor capacity were also improved by Tim-3 antibody injection (Figure S3). These data showed that peripheral injection of Tim-3 antibody inhibited viral replication and attenuated the symptoms of VSV encephalitis.

\section{Peripheral Injection of Tim-3 Antibody Enhances the Recruitment of PMDM and T Cells Into the Brain}

Because of cytokine and chemokine secretion, viral infection of the CNS may result in the recruitment of innate and adaptive immune cells to the brain. To determine the mechanisms of Tim3 antibody-mediated protection against VSV encephalitis, we first examined whether there was enhanced immune cell recruitment following antibody injection. The data in Figure 2 show that $\mathrm{CD}^{+} \mathrm{T}$ cells, $\mathrm{CD} 4^{+} \mathrm{T}$ cells, and $\mathrm{CD} 11 \mathrm{~b}^{+} \mathrm{CD} 45^{\text {hi }}$ cells, which are pMDM, were all markedly increased. The recruitment of peripheral immune cells to the brain is associated with the induction of chemokines (29). We also examined the expression of chemokines within the brain and chemokine receptors on $\mathrm{T}$ cells and macrophages in the presence or absence of Tim-3 antibody injection. Our data showed that peripheral injection of Tim-3 antibody increased the expression of C-C motif chemokine ligand 3 (CCL3) and $\mathrm{C}-\mathrm{C}$ motif chemokine ligand 5 (CCL5) within the brain, and of $\mathrm{C}$ - $\mathrm{C}$ chemokine receptor type 5 
A

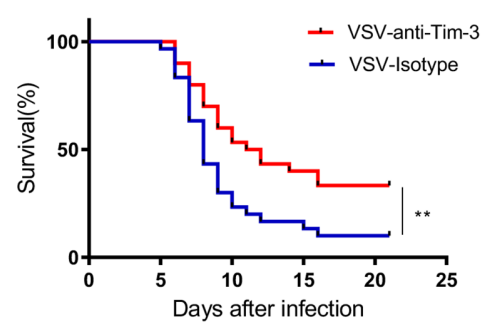

D

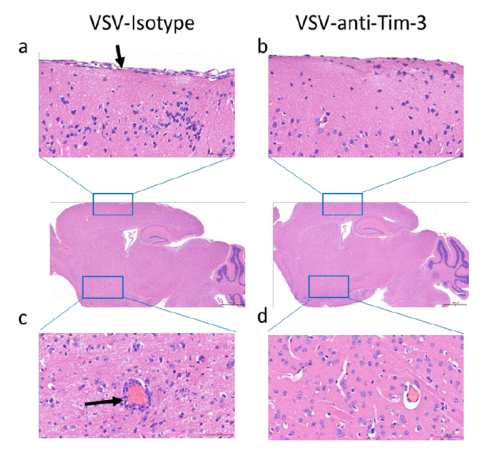

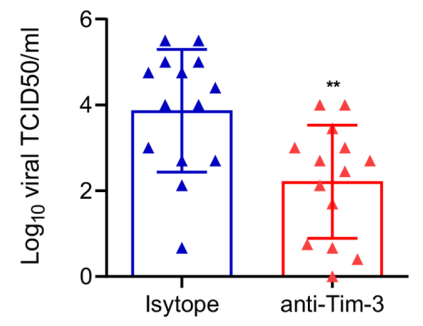

E

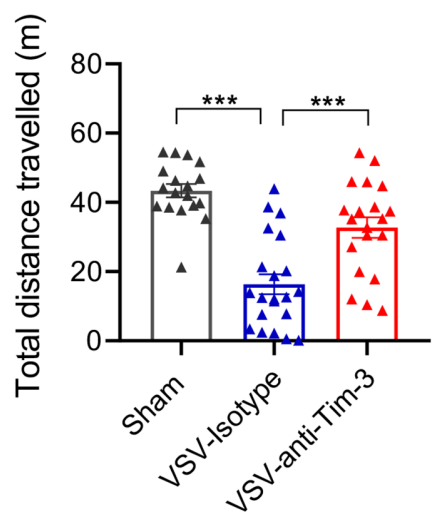

C

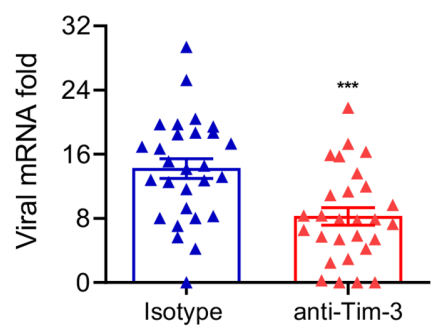

$\mathbf{F}$

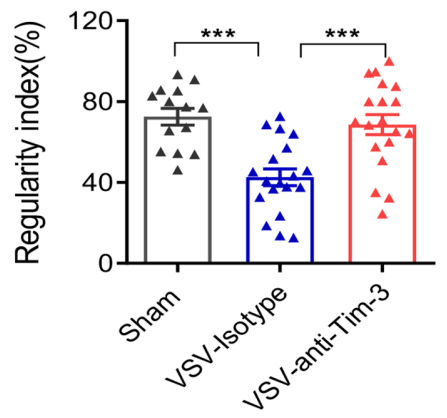

FIGURE 1 | Peripheral injection of Tim-3 antibody attenuates VSV encephalitis in mice. (A) C57BL/6 mice were intracranially injected with VSV $\left(1 \times 10^{6}\right.$ pfu/g) on day 0 and intraperitoneally injected with Tim-3 antibody (10 mg/kg, $n=15)$ or isotype control $(n=15)$ on days $-2,-1,1$, and 2. Survival rate was analyzed. (B-D) Mice were infected and treated as elaborated in (A). Brain tissues were collected on day 5 post-infection and VSV loads were analyzed by TCID50 assay (B, n=14 in each group). VSV replication were analyzed by real-time polymerase chain reaction (C, $n=28$ in each group), and (D) Hematoxylin and eosin stain of the brain section. Arrow in a) shows the damaged meningitis which is absent in b). Arrow in c) shows perivascular lymphocyte cuffing which is a well-known indicator of inflammation in the brain. No perivascular lymphocyte cuffing was found in d). The data shown are representative of six samples from each group. Original magnification $\times 100$. (E) Mice underwent the open-field test, and their spontaneous locomotor activity was evaluated by measuring the distance traveled in a defined area for 10 min. (F) Mice underwent the CatWalk analysis assay to evaluate locomotor deficits via measuring the regularity index with a maximum of $10 \mathrm{~s}$ permissible to traverse a glass plate. In (B, C, E, F), the data are expressed as mean \pm SEM of three independent experiments. ${ }^{\star \star} \mathrm{p}<0.01,{ }^{\star \star \star} \mathrm{p}<0.001$.

(CCR5) and C-X-C chemokine receptor 3 (CXCR3) on the macrophages and $\mathrm{CD}^{+} \mathrm{T}$ cells, respectively (Figure S4). These data showed that Tim-3 antibody injection enhanced the recruitment of peripheral immune cells by increasing chemokine/chemokine receptor expression.

\section{Depletion of Macrophages Abolished Tim-3 Antibody-Mediated Protection Against VSV Encephalitis}

We have previously found that Tim-3 blockade enhances macrophage activation $(23,24)$, and another study has shown that peripheral monocytes/macrophages play a protective role in the immune response against viral encephalitis (30). To determine whether macrophages account for Tim-3 antibodymediated protection against VSV encephalitis, Clodronate Liposomes were used to deplete the monocytes/macrophages. In murine spleen, Clodronate Liposomes had an efficient depletion on macrophages (Figures S6A, B). In Figures 3A, B show that when macrophages were depleted, VSV infectioninduced and Tim-3 antibody-enhanced macrophage recruitment disappeared. More importantly, macrophage depletion also abolished Tim-3 antibody-mediated suppression of virus replication (Figure 3C). Tim-3 antibody improved neuroethology as evidenced by comparable locomotor activity (Figure 3D) and locomotor deficits (Figure 3E) than that in the isotype control group. These data suggest that macrophages play a critical role in Tim-3 antibody-mediated protection against VSV encephalitis.

\section{Peripheral Injection of Tim-3 Antibody Promotes the Activation of VSV-Specific $\mathrm{CD8}^{+} \mathrm{T}$ Cells and Enhances the Expression of MHC-I on Macrophages/ Microglia}

Virus-specific $\mathrm{CD}^{+} \mathrm{T}$ cell activation plays a critical role in antiviral immunity. Here, we first examined whether Tim-3 antibody injection promoted VSV-specific $\mathrm{CD}^{+} \mathrm{T}$ cell activation by using VSV-peptide/MHC-I tetramer staining in a flow cytometry assay. The data in Figure $\mathbf{4 A}$ show that the percentage of VSV-specific $\mathrm{CD}^{+}{ }^{+} \mathrm{T}$ cells was markedly increased within the brain tissues from Tim-3 antibody-treated mice than 
in those from the control mice. In addition, when the brain tissues were stained with immunofluorescent labels of CD8, MHC-I-VSV-tetramer, and 4',6-diamidino-2-phenylindole (DAPI), the data confirmed the enhanced VSV-specific $\mathrm{CD}^{+}$ T cell activation in Tim-3 antibody-treated mice (Figure 4B).

MHC-I-mediated viral antigen presentation is a key process involved in the activation of $\mathrm{CD} 8^{+} \mathrm{T}$ cells. Many viruses escape immune attack by restricting the surface expression and half-life of MHC-I (31), which reflects the critical role of MHC-I in antiviral immunity. To determine the mechanism by which Tim3 antibody enhanced $\mathrm{CD}^{+}{ }^{+} \mathrm{T}$ cell activation, we examined the expression of MHC-I on peripheral and recruited macrophages following Tim-3 antibody injection. The data in Figure $\mathbf{5}$ show that the median fluorescence intensity of MHC-I was upregulated on $\mathrm{F} 4 / 80^{+}$peripheral macrophages, $\mathrm{CD}_{11} \mathrm{~b}^{+} \mathrm{CD} 45^{\mathrm{hi}} \mathrm{pMDM}$, and $\mathrm{CD} 11 \mathrm{~b}^{+} \mathrm{CD} 45^{\text {Low }}$ microglia in mice that received Tim-3 antibody. These data suggested that Tim-3 antibody enhanced antiviral immunity by promoting MHC-I-mediated antigen presentation and VSV-specific CD8 ${ }^{+}$ $\mathrm{T}$ cell activation.

\section{Tim-3 Blockade Increases MHC-I Expression in Macrophages by Inhibiting MARCH9-Mediated Proteasomal Degradation of MHC-I}

We then investigated the mechanism by which Tim-3 blockade enhanced MHC-I expression. Surface expression and half-life of MHC-I (the molecule responsible for $\mathrm{CD}^{+} \mathrm{T}$ cell antigen presentation and initiation of adaptive immunity) are controlled via ubiquitination. Here, we observed for the first time that Tim-3 was involved in the post-translational modification of MHC-I. The data in Figures 6A, B show that when Tim-3 was overexpressed, blocking protein synthesis by CHX led to a decreased MHC-I protein expression; whereas, addition of MG132, a proteasome inhibitor, abolished Tim-3 overexpression-mediated MHC-I decrease. These data show that Tim-3 promoted proteasome-dependent degradation of MHC-I.

Ubiquitination is one of the most versatile post-translational modifications that is indispensable for antiviral immunity, and ubiquitination of MHC-I has been widely investigated recently (32). Next, we tested whether Tim-3 promoted MHC-I

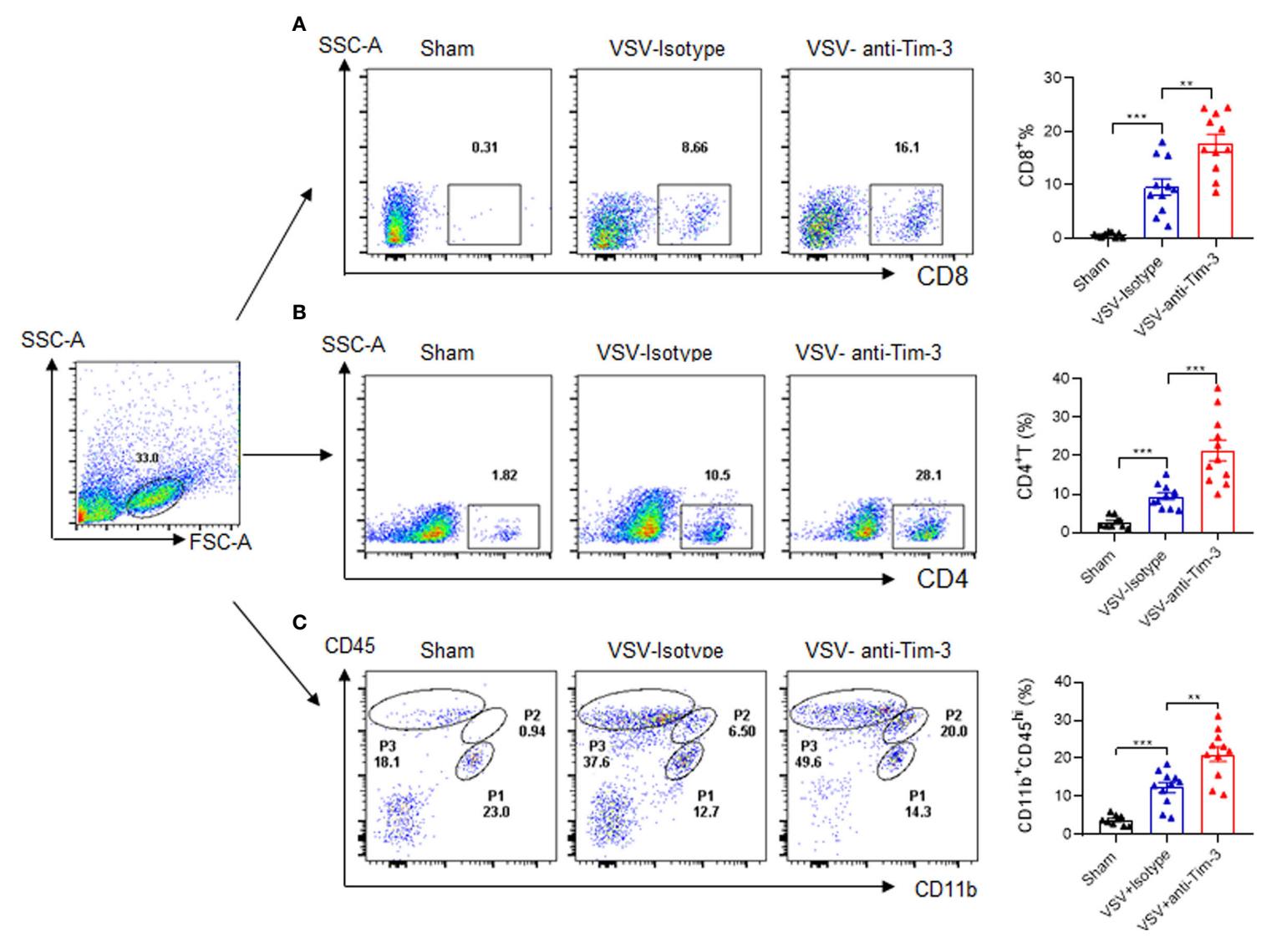

FIGURE 2 | Peripheral injection of Tim-3 antibody enhances the recruitment of peripheral immune cells. Mice were treated as shown in Figure 1. On day 5 postinfection, mice were sacrificed, brain tissues were collected from them, and single-cell suspensions were prepared as described in the Material and Methods. The infiltration of $\mathrm{CD}^{+} \mathrm{T}$ cells $\mathbf{( A )}$, CD8 ${ }^{+} \mathrm{T}$ cells $\mathbf{( B )}$ and peripheral monocyte-derived macrophages (pMDM) cells (CD11 ${ }^{+} \mathrm{CD} 45^{\text {hi }}$ ) (C) were examined by flow cytometry analysis. The left panel shows the representative fluorescence-activated cell sorting (FACS) plot, and the right panel are presented as means \pm SEM of three independent experiments. ${ }^{* \star} \mathrm{p}<0.01,{ }^{\star * \star} \mathrm{p}<0.001$. 
A

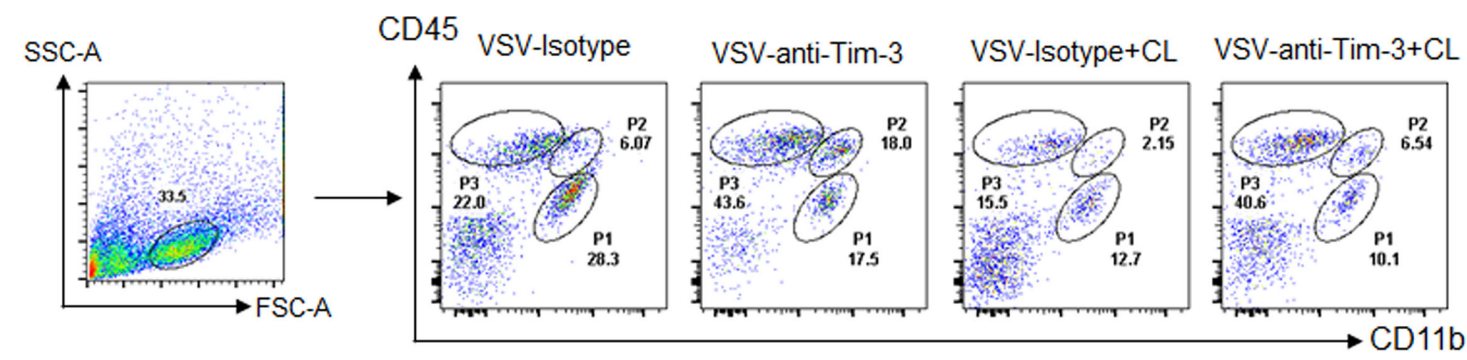

B

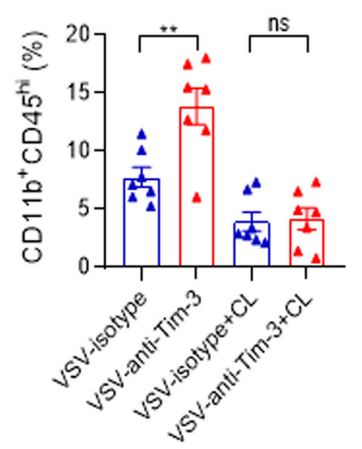

C

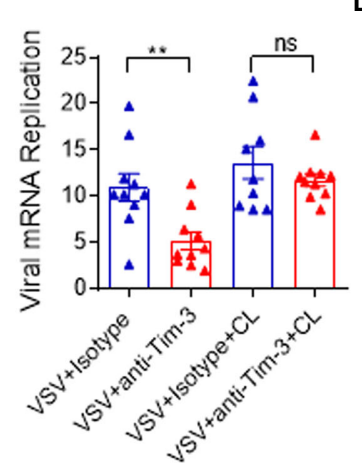

D

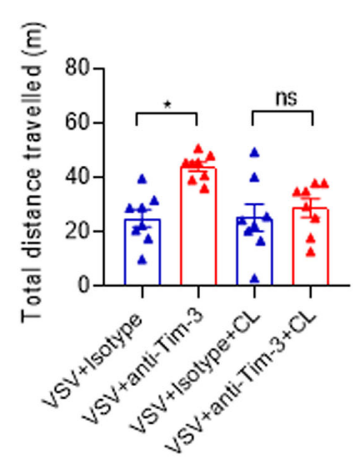

E

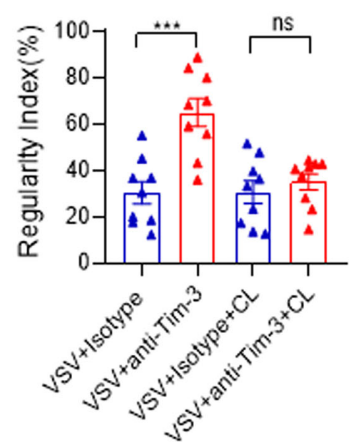

FIGURE 3 | Deletion of macrophages abolished Tim-3 antibody-mediated immune protection against VSV encephalitis. Mice were treated as shown in Figure 1, and untreated mice were used as sham controls. For macrophage depletion, Clodronate Liposomes (CL) were intravenously injected three times on days -2, 0, and 2. On day 5 post-infection, the mice were sacrificed. Brain tissues were collected, and single-cell suspensions were prepared and stained for detecting CD11b and CD45 expression by flow cytometry analysis. (A) shows the representative fluorescence-activated cell sorting (FACS) plot; while, (B) shows the mean \pm SEM of three independent experiments. (C) The brain tissues were analyzed for VSV replication by real-time polymerase chain reaction, and the results are expressed as mean \pm SEM of three independent experiments. (D) Mice were subjected to the open-field test to evaluate spontaneous locomotor activity by measuring the distance traveled in a defined area for $10 \mathrm{~min}$. Data are expressed as mean \pm SEM of three independent experiments. (E) Mice were subjected to the CatWalk analysis to evaluate locomotor deficits by measuring the regularity index, with maximum $10 \mathrm{~s}$ to traverse a glass plate. Data are expressed as mean \pm SEM of three independent experiments. ns, not significant, ${ }^{\star} p<0.05,{ }^{\star \star} p<0.01,{ }^{\star \star *} p<0.001$.

degradation via ubiquitination modification. The data in Figure 6C show that co-transfection with Tim-3 significantly enhanced the ubiquitination of MHC-I. Next, possible E3 ligases were identified. Several MARCH family proteins, including MARCH2, MARCH9, and MARCH10 have been found to be associated with the ubiquitination modification of MHC-I. We first examined whether Tim-3 regulated the protein expression of MARCH. The results showed that in RAW264.7 cells silenced with Tim-3 or treated with Tim-3 blocking antibody, the expression of MARCH9 was decreased (Figure S5). Other MARCH proteins, including MARCH2 and MARCH10, were not significantly altered when Tim-3 signaling was blocked (data not shown). To test whether MARCH9 was involved in Tim-3mediated ubiquitination and degradation of MHC-I, we cotransfected K48-linked ubiquitin, MARCH9, and Tim-3 into HEK293T cells. The data in Figures 6D, E show that protein levels of MHC-I were significantly decreased in the presence of MARCH9 and increased dose of Tim-3. We then examined which kind of ubiquitination was involved in Tim-3 mediated MHC-I degradation. As K48- linked ubiquitination leads to proteasomal dependent degradation (33), to test whether Tim3 enhances the K48-linked ubiquitination, here we co-transfected plasmids encoding ubiquitin-K48, MARCH9 and Tim-3 into HEK293T cells. The data in Figure 6F showed a marked increase in MHC-I ubiquitination in the presence of MARCH9 and Tim3. These data showed that Tim-3 signaling promotes proteasomal degradation of MHC-I via MARCH9 and explained how Tim-3 blockade using antibodies enhanced MHC-I-mediated antigen presentation in VSV encephalitis.

A schematic diagram of how Tim-3 antibody augments peripheral immune cells to combat viral encephalitis is shown in Figure 7.

\section{DISCUSSION}

Viral encephalitis is a devastating disease with no specific treatment other than supportive care. Host immune responses play a critical role in fighting against the invading virus while maintaining neural homeostasis. Thus, methods that can be employed to appropriately manipulate the immune response during viral encephalitis are urgently needed. Here, we identified a novel mode of combating VSV encephalitis. Peripheral injection of Tim-3 antibody markedly decreased the 
A
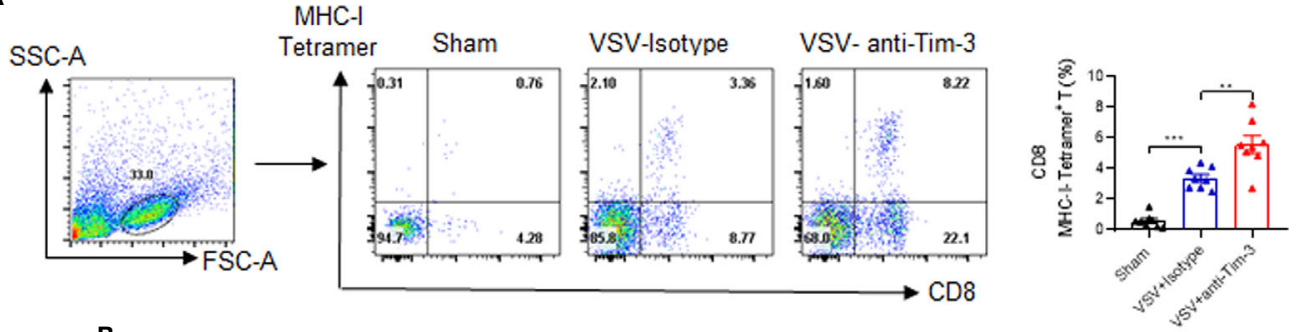

B

DAPI
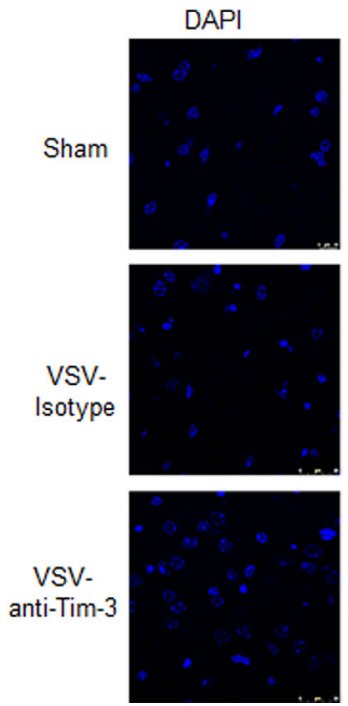

CD8
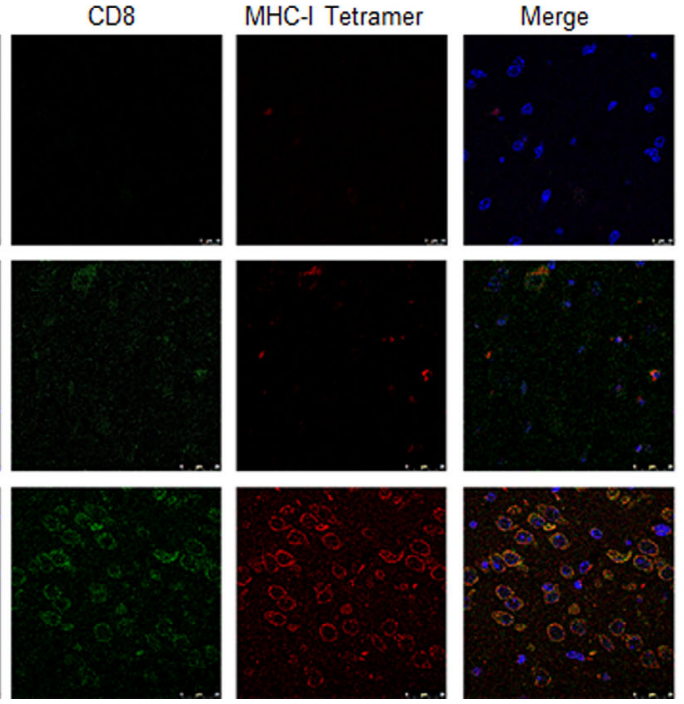

FIGURE 4 | Peripheral injection of Tim-3 antibody enhances the activation of VSV-specific CD8 ${ }^{+}$T cells. Mice were treated as described in Figure 1. On day 5 post-infection, brain tissues were collected. (A) Single-cell suspensions were prepared and stained with antibodies against CD8 and MHC-I-VSV-tetramer. Left panel shows the representative fluorescence-activated cell sorting (FACS) plot and right panel shows the mean percentage \pm SEM of three independent experiments. ${ }^{\star \star} \mathrm{P}<0.01,{ }^{\star \star \star} \mathrm{P}<0.001$. (B) The brain tissues were stained with immunofluorescence labels for CD8 (green), MHC-I-VSV-tetramer (red), and DAPI (blue). Data shown are representative images of three independent experiments.

viral load, tissue damage, and mortality associated with VSV encephalitis. Investigation of the underlying mechanism revealed that Tim-3 blockade augmented the systemic expression of interferons, enhanced the recruitment of macrophages and $\mathrm{T}$ cells into the brain, and increased the expression and antigen presentation activity of MHC-I on macrophages. Importantly, we observed a novel mechanism by which Tim-3 signaling regulated MHC-I expression; that is, by promoting proteasome-dependent degradation of MHC-I via E3 ligase MARCH9. Thus, our data shed light on the cross-talk between the peripheral immune response and CNS; consequently, we have discovered a novel mechanism that possibly augments their coordination to fight against viral encephalitis.

The cross-talk between the CNS and peripheral immune system under different physio-pathological conditions remains largely unclear. Neuroinflammation and neuroinfection can alter the $\mathrm{BBB}$; however, whether this alteration is beneficial or not depends on different conditions. Further, there are controversial reports concerning whether the augmented immune response plays a protective or pathogenic role during neuroinfection and neuroinflammation (34). However, as there is no doubt that tightly regulated immune responses do play a critical role in infection control, it is urgent to identify ways to manipulate the immune response individually or to a proper state during viral encephalitis. In animal models of Japanese encephalitis, the BBB breaks down following infection, which results in a large influx of monocytes and lymphocytes into the brain $(35,36)$. Notably, the presence of $\mathrm{CD}^{+} \mathrm{T}$ cells and response of type I interferons are negatively correlated with the viral load; whereas, $\mathrm{CD}^{+} \mathrm{T}$ cells and innate immune responses are pathogenic (37). These studies provided possible targets, such as $\mathrm{CD}^{+} \mathrm{T}$ cells and interferons, that may be used to manipulate the immune response against viral encephalitis. Meanwhile, another study has shown that clodronate liposome-mediated depletion of monocytes/ macrophages results in increased mortality, thereby indicating that hematogenously derived monocytes/macrophages are required for immune protection against viruses (38). These studies support our findings as they show that augmented recruitment of macrophages and $\mathrm{T}$ cells plays a protective role. During neuroinflammation, such as in Alzheimer's disease, revitalizing brain immunity by recruiting peripheral myeloid cells and decreasing local immunosuppressive myeloid 
A
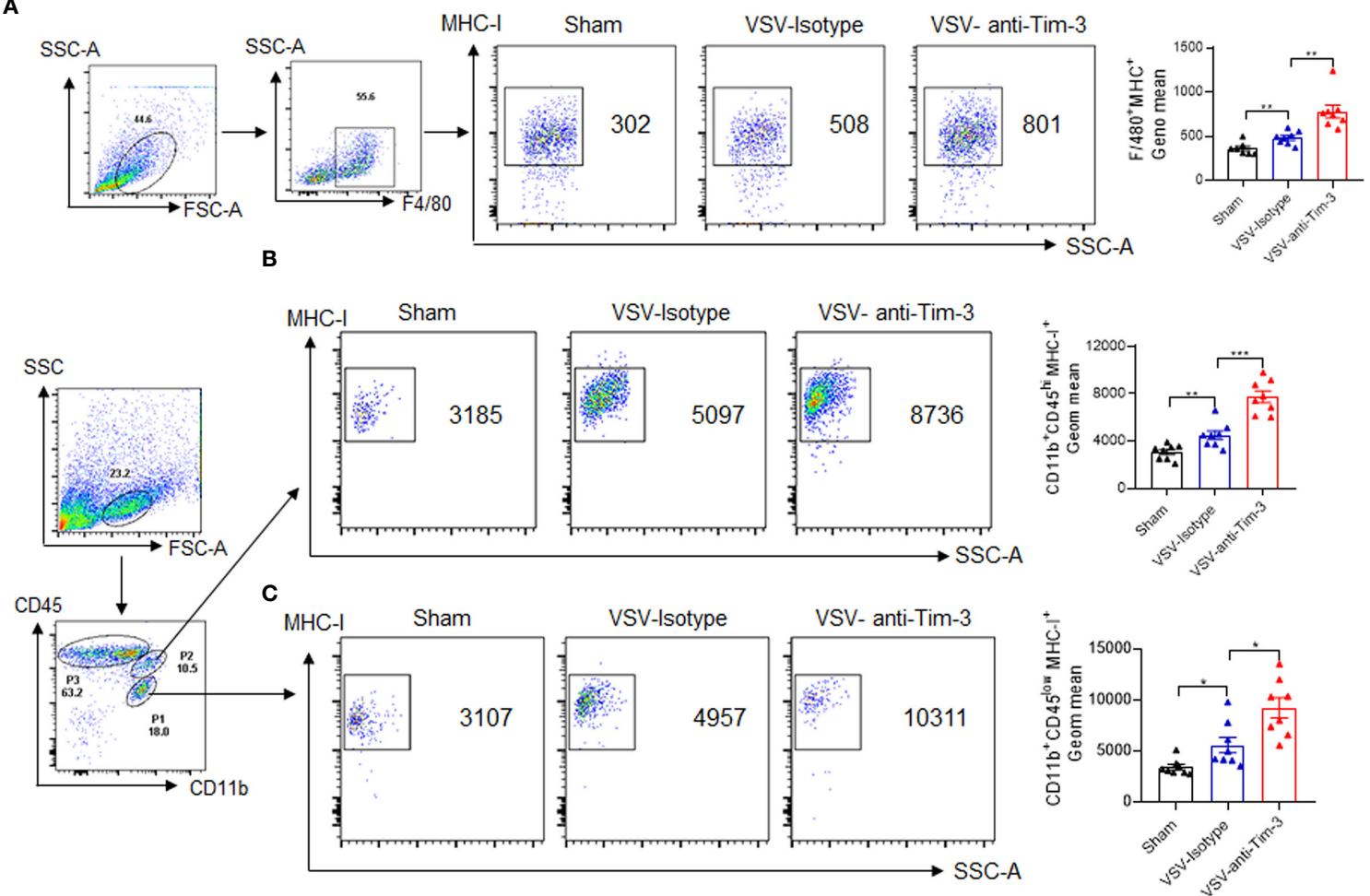

FIGURE 5 | Peripheral injection of Tim-3 antibody enhances the expression of major histocompatibility complex-I (MHC-I). Mice were treated as shown in Figure $\mathbf{1}$. On day 5 post-infection, the mice were sacrificed. (A) Peritoneal macrophages were collected and stained with antibodies against F4/80 and MHC-I. The left panel shows the representative fluorescence-activated cell sorting (FACS) plot and right panel shows the mean fluorescence intensity (MFI) \pm SEM of three independent experiments. (B, C) Brain tissues were collected, and single-cell suspensions were prepared and stained with anti-CD11b and anti-CD45 antibody. CD11b ${ }^{+} C D 45^{\text {hi }}$ pMDM (P2) and CD11 b ${ }^{+} \mathrm{CD} 45^{\mathrm{Low}}$ microglia (P1) were further gated to analyze the fluorescence intensity of MHC-I using MHC-I antibody. The left panel shows the representative FACS plots and the right panel shows the MFI \pm SEM of three independent experiments. ${ }^{\star} p<0.05,{ }^{\star \star} p<0.01,{ }^{\star \star *} p<0.001$.

population is a novel therapeutic approach, which in turn supports the beneficial roles of the peripheral immune system (19). Thus, manipulating the immune response to a proper state is necessary for controlling different diseases. Here, we observed that enhanced $\mathrm{CD}^{+} \mathrm{T}$ cell activation following Tim-3 blockade played a protective role in virus control, and this finding is consistent with other studies that have demonstrated the protective role of $\mathrm{CD}^{+} \mathrm{T}$ cells in herpes simplex virus-1 encephalitis (39). The versatility of Tim-3 blockade in protection against other viral encephalitis will be investigated in future studies.

Here the antibody of Tim-3 is administrated before and after virus infection. We also tested the efficiency of anti-Tim-3 antibody on VSV encephalitis by injecting the antibody only after virus infection. The data in Figure S6 showed that injecting the antibody only after virus infection could moderately, but not significantly, decrease the survival rate of the infected mice. However, it could significantly decrease the virus load and improve mice behavior in open field test, suggesting that injecting the antibody only after virus infection also provides protection against VSV encephalitis. Here we focus on exploring the mechanisms of anti-Tim-3 antibody injection before and after virus infection mediated protection on VSV encephalitis.
Although the strategy in detail may be improved in the further, here we demonstrate the therapeutical potential of anti-Tim-3 antibody in virus encephalitis. VSV replication is very sensitive to type I interferons (IFN-I) signaling. As our data in Figure S2 showed that anti-Tim-3 antibody increases the expression of interferons in mice in vivo, it is interesting to know whether antiTim-3 antibody mediated protection against VSV encephalitis depends on interferons. Our data in Figure 3 showed that macrophages depletion with Clodronate Liposomes abolished Tim-3 antibody mediated protection. However, macrophages depletion with CL did not markedly alter the expression of interferons (Figure S7). These data suggested that the Tim-3 antibody mediated protection depends on macrophages rather than the increased interferons.

MHC-I-mediated antigen presentation and $\mathrm{CD}^{+} \mathrm{T}$ cell activation play critical roles in antiviral immunity. MHC-I expression can be regulated at both the mRNA and protein levels. In this study, we observed that peripheral injection of Tim-3 antibody systemically increased the expression of interferons, which possibly indirectly increases the transcription of MHC-I (31). Previously, we found that Tim3 signaling may inhibit MHC-I transcription via STAT1 (40). Another study reported that viruses suppress MHC-I 


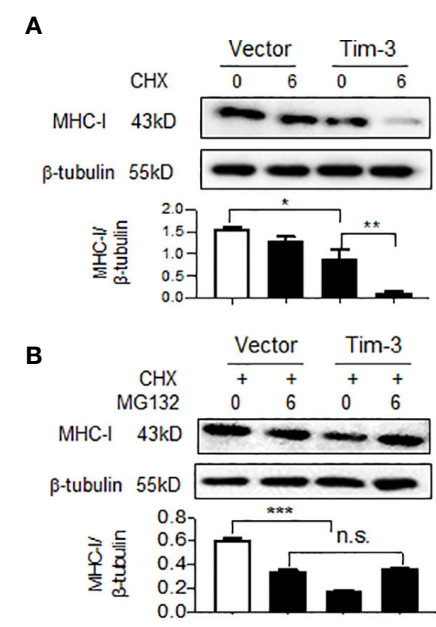

C

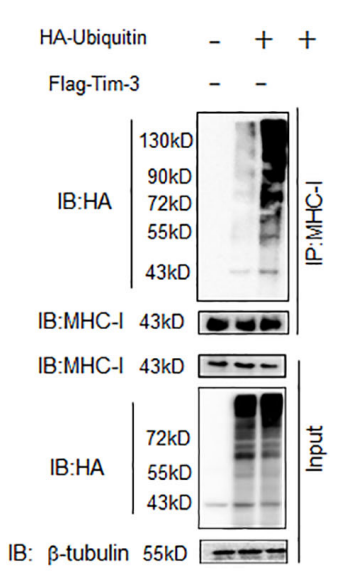

D

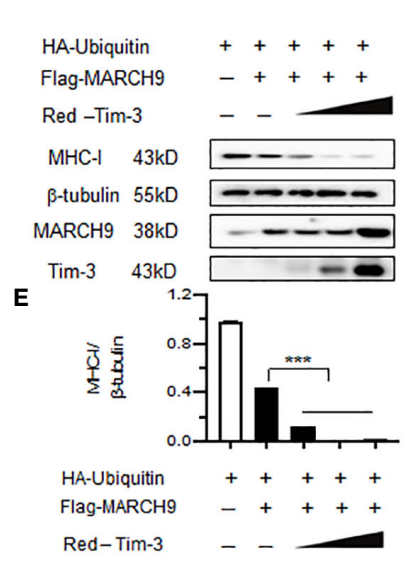

$\mathbf{F}$

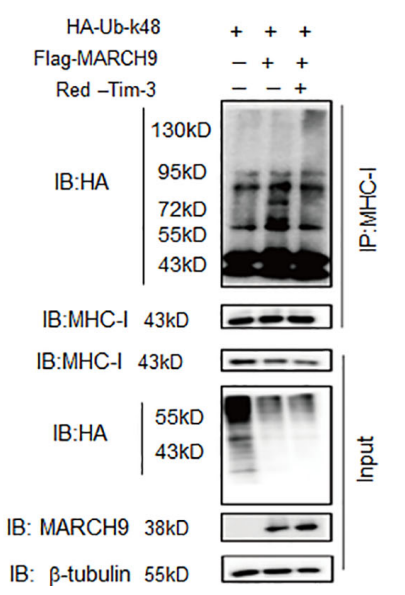

FIGURE 6 | Tim-3 promotes proteasome-dependent degradation and ubiquitination of major histocompatibility complex-I (MHC-I). (A) The HEK293T cells were transfected with plasmids encoding Flag-Tim-3 or vector control. Cells were then treated with or without a protein synthesis inhibitor CHX (10 $\mu \mathrm{M})$ for 6 h. Twentyfour hours following transfection, cells were harvested and the level of $\mathrm{MHC}-\mathrm{I}$ protein was examined by western blot. The upper panel shows the representative image of three independent experiments and the down panel shows the value of the MHC-1/ $\beta$-tubulin protein ratio which is expressed as mean \pm SEM of three independent experiments. (B) The HEK293T cells were transfected with plasmids encoding Flag-Tim-3 or vector control. All cells were treated with $\mathrm{CHX}(10 \mu \mathrm{M})$ in the presence or absence of the proteasome inhibitor MG132 $(20 \mu \mathrm{g} / \mathrm{ml})$ for $6 \mathrm{~h}$. Twenty-four hours following transfection, cells were harvested and the level of MHC-I protein was examined by western blot. The upper panel shows the representative image of three independent experiments and the down panel shows the value of the MHC-1/ $/$-tubulin protein ratio which is expressed as mean \pm SEM of three independent experiments. (C) Plasmid encoding HA-Tim-3 was transfected into HEK293T cells for 24h, followed by immunoprecipitation with antibody to MHC-I and then Western blot analysis of MHC-I ubiquitination with antibody to HA. The data shown are the representative image for those of three independent experiments. (D\&E) The HEK293T cells were transfected with plasmids encoding HAubiquitin, Flag-MARCH9 and increasing doses of a plasmid encoding Red-Tim-3 (0.5,1, and $2 \mu \mathrm{g})$. The level of MHC-I protein was examined in cells harvested 24h later. (D) shows the representative image of three independent experiments and (E) shows the value of the MHC-1/ $\beta$-tubulin protein ratio which is expressed as mean \pm SEM of three independent experiments. (F) Plasmids encoding HA-tag-Ubiquitin K48 (K48-lined ubiquitination leads to proteasomal-mediated degradation of the target protein), Flag-MARCH9, and Red-Tim-3 were transfected into HEK293T cells. Cells were treated with MG132 (20 $\mu \mathrm{g} / \mathrm{ml})$ for $6 \mathrm{~h}$ before harvesting protein lysates, followed by immunoprecipitation with antibody to MHC-I and then Western Blot analysis of K48-linked MHC-I ubiquitination with antibody to HA. These experiments were repeated 3 times with similar results. In A\&B, the data shown are expressed as mean \pm SEM of three independent experiments. ${ }^{* \star} \mathrm{p}<0.01 ;{ }^{* *} \mathrm{p}<0.001$.

transcription in vivo, which might limit $\mathrm{T}$ cell-mediated killing of rotavirus-infected enterocytes $(41,42)$. Compared to the regulation at the transcriptional level, post-translational modification of MHC-I, which markedly affects the half-life of MHC-I, provides a more rapid control of antigen presentation (43). The ability of ubiquitin to control membrane protein localization and expression has significant consequences for cellular functions. Ubiquitination plays important regulatory roles in the immune system (44), and ubiquitination of MHC-I was first described in the context of viral proteins that target MHC-I for degradation in the endoplasmic reticulum and at the cell surface. Ubiquitination of MHC-I, MHC-II, and CD1a by different members of the MARCH family (32) of E3 ubiquitin ligases is a key event in the regulation of potent immunostimulatory properties of activated innate immune cells (45). Epstein-Barr virus inhibits MHC-I expression by regulating ubiquitination (46). Here, we identified a new post-translational mechanism of MHC-I that was mediated by Tim-3. By enhancing the expression of MARCH9, Tim-3 promoted the ubiquitination and degradation of MHC-I. These data explain the mechanism by which Tim-3 antibody injection enhanced MHC-I expression and antigen presentation in macrophages.
Recently, checkpoint inhibitors PD-1 and CTLA-4 have been used as therapeutic targets for immune disorders, tumors (47), autoimmune diseases (20) and neuroinflammatory diseases such as Alzheimer's disease (19). Tim-3 is considered as the nextgeneration checkpoint inhibitor, and its antagonist has been widely investigated (48). We have previously focused on the role of Tim-3 in maintaining the homeostasis of innate immunity and found that Tim-3 inhibits TLR4-induced macrophage activation via the NF- $\kappa B$ signaling pathway (49), and inhibits M1 macrophage polarization via STAT1 (24). Macrophages also play critical roles in antiviral immunity both as an innate immune player and as antigen-presenting cells. In this study, we found that Tim-3 blockade using an antibody augmented the systemic immune response marked by increased expression of chemokines and their receptors, and as a result, enhanced the recruitment of infiltrating $\mathrm{CD} 11 \mathrm{~b}^{+} \mathrm{CD} 45^{\mathrm{hi}} \mathrm{pMDM}$ into the brain, and more importantly, enhanced the antigenpresenting activity of the macrophages. The depletion of macrophages abolished Tim-3 antibody-mediated protection against VSV encephalitis, suggesting that macrophages play a critical role in Tim-3 blockade-mediated protection.

In summary, we identified a novel method that may be used to manipulate the immune response to combat viral encephalitis. 


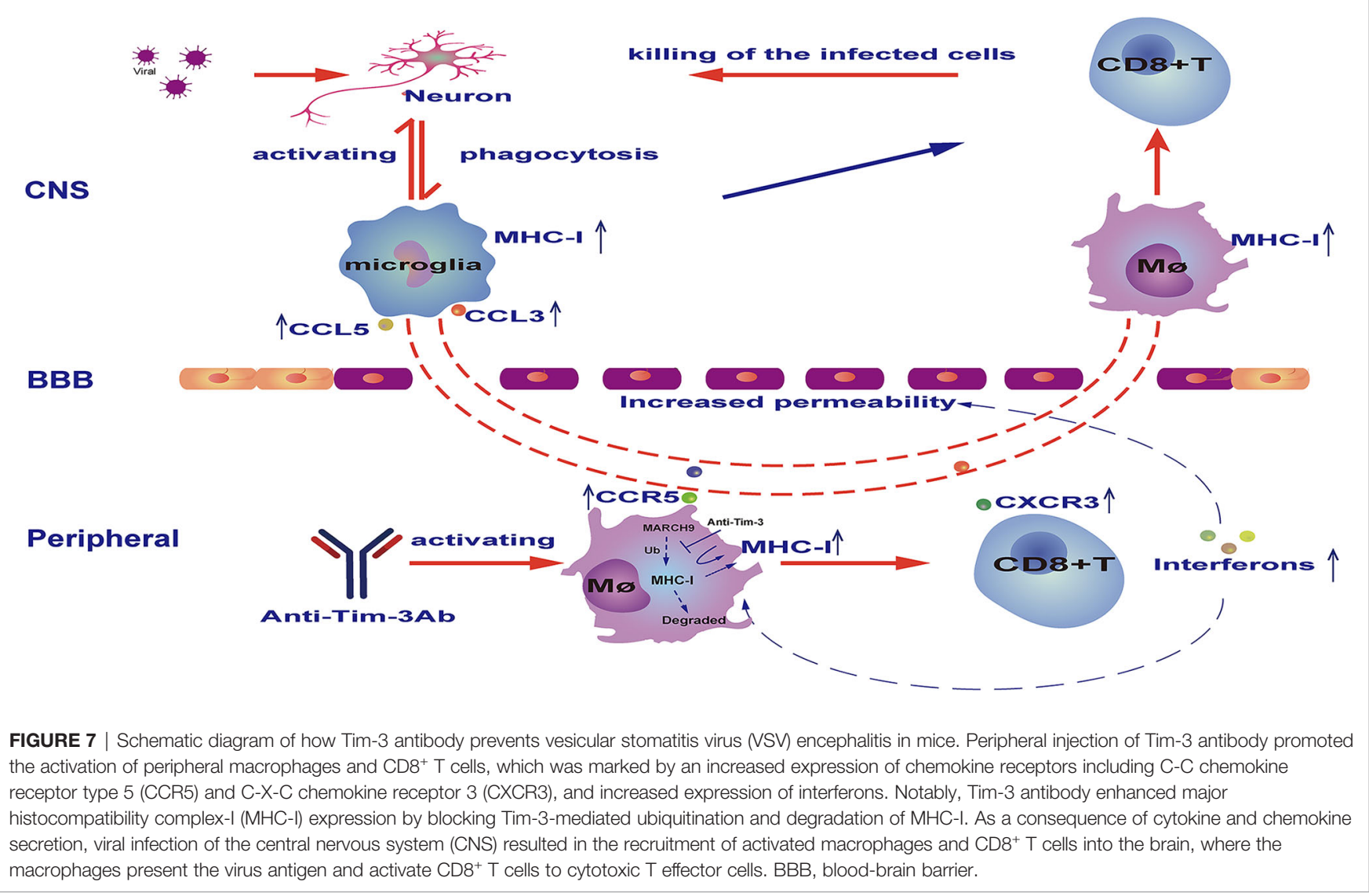

Like PD-1, which can be used to attenuate neuroinflammation in Alzheimer's disease by augmenting the systemic immune response, Tim-3 can be used to inhibit neuroinfection in VSV encephalitis by systemically augmenting the immune responses. Here, we identified that Tim-3 controlled the response of macrophages by enhancing the ubiquitination and degradation of MHC-I, and Tim-3 negatively regulated the macrophagemediated antigen presentation. Our findings provide insight into the underlying mechanism of immune response against viral encephalitis and may have potential therapeutic applications.

\section{DATA AVAILABILITY STATEMENT}

The original contributions presented in the study are included in the article/Supplementary Material. Further inquiries can be directed to the corresponding authors.

\section{ETHICS STATEMENT}

The animal study was reviewed and approved by Beijing Institute of Basic Medical Sciences.

\section{AUTHOR CONTRIBUTIONS}

Conception and design of the study: GH, GL, RW. Acquisition, analysis, and interpretation of data: GL, LT, CH, ZW, YG, JD. Contribution of administrative, experimental, analytic, or material support: SD, RM, YH, JD, JZ, BS. Writing-Original Draft Preparation: GL, RW. Writing-Review and Editing: GH. All authors contributed to the article and approved the submitted version.

\section{FUNDING}

This work was supported by the National Natural Sciences Foundation of China (grant nos. 81971473, 81771684) and the Beijing Natural Sciences Foundation (grant no.7192145).

\section{SUPPLEMENTARY MATERIAL}

The Supplementary Material for this article can be found online at: https:/www.frontiersin.org/articles/10.3389/fimmu.2021.667478/ full\#supplementary-material 


\section{REFERENCES}

1. Fooks AR, Banyard AC, Horton DL, Johnson N, McElhinney LM, Jackson AC. Current Status of Rabies and Prospects for Elimination. Lancet (2014) 384:1389-99. doi: 10.1016/S0140-6736(13)62707-5

2. Labeaud AD, Bashir F, King CH. Measuring the Burden of Arboviral Diseases: The Spectrum of Morbidity and Mortality From Four Prevalent Infections. Popul Health Metr (2011) 9:1. doi: 10.1186/1478-7954-9-1

3. Venkatesan A, Michael BD, Probasco JC, Geocadin RG, Solomon T. Acute Encephalitis in Immunocompetent Adults. Lancet (2019) 393:702-16. doi: 10.1016/S0140-6736(18)32526-1

4. Domingues RB. Viral Encephalitis: Current Treatments and Future Perspectives. Cent Nerv Syst Agents Med Chem (2012) 12:277-85. doi: $10.2174 / 187152412803760582$

5. Waltl I, Kaufer C, Gerhauser I, Chhatbar C, Ghita L, Kalinke U, et al. Microglia Have a Protective Role in Viral Encephalitis-Induced Seizure Development and Hippocampal Damage. Brain Behav Immun (2018) 74:186-204. doi: 10.1016/j.bbi.2018.09.006

6. Daneman R, Prat A. The Blood-Brain Barrier. Cold Spring Harb Perspect Biol (2015) 7:a020412. doi: 10.1101/cshperspect.a020412

7. Merlini M, Kirabali T, Kulic L, Nitsch RM, Ferretti MT. Extravascular Cd3+ T Cells in Brains of Alzheimer Disease Patients Correlate With Tau But Not With Amyloid Pathology: An Immunohistochemical Study. Neurodegener Dis (2018) 18:49-56. doi: 10.1159/000486200

8. Hickman SE, Kingery ND, Ohsumi TK, Borowsky ML, Wang LC, Means TK, et al. The Microglial Sensome Revealed by Direct RNA Sequencing. Nat Neurosci (2013) 16:1896-905. doi: 10.1038/nn.3554

9. Ginhoux F, Greter M, Leboeuf M, Nandi S, See P, Gokhan S, et al. Fate Mapping Analysis Reveals That Adult Microglia Derive From Primitive Macrophages. Science (2010) 330:841-5. doi: 10.1126/science.1194637

10. Gomez Perdiguero E, Klapproth K, Schulz C, Busch K, Azzoni E, Crozet L, et al. Tissue-Resident Macrophages Originate From Yolk-Sac-Derived Erythro-Myeloid Progenitors. Nature (2015) 518:547-51. doi: 10.1038/ nature 13989

11. Nelson LH, Lenz KM. Microglia Depletion in Early Life Programs Persistent Changes in Social, Mood-Related, and Locomotor Behavior in Male and Female Rats. Behav Brain Res (2017) 316:279-93. doi: 10.1016/ j.bbr.2016.09.006

12. Nimmerjahn A, Kirchhoff F, Helmchen F. Resting Microglial Cells are Highly Dynamic Surveillants of Brain Parenchyma In Vivo. Science (2005) 308:13148. doi: 10.1126/science.1110647

13. Paolicelli RC, Bolasco G, Pagani F, Maggi L, Scianni M, Panzanelli P, et al. Synaptic Pruning by Microglia is Necessary for Normal Brain Development. Science (2011) 333:1456-8. doi: 10.1126/science.1202529

14. Schafer DP, Lehrman EK, Kautzman AG, Koyama R, Mardinly AR, Yamasaki R, et al. Microglia Sculpt Postnatal Neural Circuits in an Activity and ComplementDependent Manner. Neuron (2012) 74:691-705. doi: 10.1016/j.neuron. 2012.03.026

15. Fehervari Z. Publisher Correction: Macrophages: Damage Control. Nat Immunol (2018) 19:898. doi: 10.1038/s41590-018-0159-2

16. Marten NW, Stohlman SA, Bergmann CC. Role of Viral Persistence in Retaining CD8(+) T Cells Within the Central Nervous System. J Virol (2000) 74:7903-10. doi: 10.1128/JVI.74.17.7903-7910.2000

17. Bergmann CC, Parra B, Hinton DR, Chandran R, Morrison M, Stohlman SA. Perforin-Mediated Effector Function Within the Central Nervous System Requires IFN-gamma-mediated MHC Up-Regulation. J Immunol (2003) 170:3204-13. doi: 10.4049/jimmunol.170.6.3204

18. Schwartz M, Baruch K. The Resolution of Neuroinflammation in Neurodegeneration: Leukocyte Recruitment Via the Choroid Plexus. EMBO $J$ (2014) 33:7-22. doi: 10.1002/embj.201386609

19. Baruch K, Deczkowska A, Rosenzweig N, Tsitsou-Kampeli A, Sharif AM, Matcovitch-Natan O, et al. PD-1 Immune Checkpoint Blockade Reduces Pathology and Improves Memory in Mouse Models of Alzheimer's Disease. Nat Med (2016) 22:135-7. doi: 10.1038/nm.4022

20. Rosenzweig N, Dvir-Szternfeld R, Tsitsou-Kampeli A, Keren-Shaul H, BenYehuda H, Weill-Raynal P, et al. Pd-1/Pd-L1 Checkpoint Blockade Harnesses Monocyte-Derived Macrophages to Combat Cognitive Impairment in a
Tauopathy Mouse Model. Nat Commun (2019) 10:465. doi: 10.1038/ s41467-019-08352-5

21. Jones RB, Ndhlovu LC, Barbour JD, Sheth PM, Jha AR, Long BR, et al. Tim-3 Expression Defines a Novel Population of Dysfunctional T Cells With Highly Elevated Frequencies in Progressive HIV-1 Infection. J Exp Med (2008) 205:2763-79. doi: 10.1084/jem.20081398

22. Monney L, Sabatos CA, Gaglia JL, Ryu A, Waldner H, Chernova T, et al. Th1specific Cell Surface Protein Tim-3 Regulates Macrophage Activation and Severity of an Autoimmune Disease. Nature (2002) 415:536-41. doi: 10.1038/ 415536a

23. Han G, Chen G, Shen B, Li Y. Tim-3: An Activation Marker and Activation Limiter of Innate Immune Cells. Front Immunol (2013) 4:449. doi: 10.3389/ fimmu.2013.00449

24. Jiang X, Zhou T, Xiao Y, Yu J, Dou S, Chen G, et al. Tim-3 Promotes TumorPromoting M2 Macrophage Polarization by Binding to STAT1 and Suppressing the STAT1-miR-155 Signaling Axis. Oncoimmunology (2016) 5:e1211219. doi: 10.1080/2162402X.2016.1211219

25. Chen W, Han C, Xie B, Hu X, Yu Q, Shi L, et al. Induction of Siglec-G by RNA Viruses Inhibits the Innate Immune Response by Promoting RIG-I Degradation. Cell (2013) 152:467-78. doi: 10.1016/j.cell.2013.01.011

26. Hosseini S, Wilk E, Michaelsen-Preusse K, Gerhauser I, Baumgartner W, Geffers R, et al. Long-Term Neuroinflammation Induced by Influenza A Virus Infection and the Impact on Hippocampal Neuron Morphology and Function. J Neurosci (2018) 38:3060-80. doi: 10.1523/JNEUROSCI.174017.2018

27. Jones KA, Maltby S, Plank MW, Kluge M, Nilsson M, Foster PS, et al. Peripheral Immune Cells Infiltrate Into Sites of Secondary Neurodegeneration After Ischemic Stroke. Brain Behav Immun (2018) 67:299-307. doi: 10.1016/j.bbi.2017.09.006

28. Pastore G, Carraro M, Pettini E, Nolfi E, Medaglini D, Ciabattini A. Optimized Protocol for the Detection of Multifunctional Epitope-Specific Cd4(+) T Cells Combining Mhc-Ii Tetramer and Intracellular Cytokine Staining Technologies. Front Immunol (2019) 10:2304. doi: 10.3389/fimmu.2019.02304

29. Prinz M, Priller J, Sisodia SS, Ransohoff RM. Heterogeneity of CNS Myeloid Cells and Their Roles in Neurodegeneration. Nat Neurosci (2011) 14:1227-35. doi: $10.1038 / \mathrm{nn} .2923$

30. Steel CD, Breving K, Tavakoli S, Kim WK, Sanford LD, Ciavarra RP. Role of Peripheral Immune Response in Microglia Activation and Regulation of Brain Chemokine and Proinflammatory Cytokine Responses Induced During VSV Encephalitis. J Neuroimmunol (2014) 267:50-60. doi: 10.1016/ j.jneuroim.2013.12.002

31. van de Weijer ML, Luteijn RD, Wiertz EJ. Viral Immune Evasion: Lessons in MHC Class I Antigen Presentation. Semin Immunol (2015) 27:125-37. doi: 10.1016/j.smim.2015.03.010

32. Gatti E. Monitoring MHC Ubiquitination by MARCH Ubiquitin Ligases. Methods Mol Biol (2019) 1988:259-70. doi: 10.1007/978-1-4939-9450-2_18

33. Ye B, Liu B, Hao L, Zhu X, Yang L, Wang S, et al. Klf4 Glutamylation is Required for Cell Reprogramming and Early Embryonic Development in Mice. Nat Commun (2018) 9:1261. doi: 10.1038/s41467-018-03008-2

34. Chhatbar C, Detje CN, Grabski E, Borst K, Spanier J, Ghita L, et al. Type I Interferon Receptor Signaling of Neurons and Astrocytes Regulates Microglia Activation During Viral Encephalitis. Cell Rep (2018) 25:118-129 e4. doi: 10.1016/j.celrep.2018.09.003

35. Han YW, Choi JY, Uyangaa E, Kim SB, Kim JH, Kim BS, et al. Distinct Dictation of Japanese Encephalitis Virus-Induced Neuroinflammation and Lethality Via Triggering TLR3 and TLR4 Signal Pathways. PloS Pathog (2014) 10:e1004319. doi: 10.1371/journal.ppat.1004319

36. Kim JH, Patil AM, Choi JY, Kim SB, Uyangaa E, Hossain FM, et al. CCL2, But Not its Receptor, is Essential to Restrict Immune Privileged Central Nervous System-Invasion of Japanese Encephalitis Virus Via Regulating Accumulation of CD11b(+) Ly-6C(hi) Monocytes. Immunology (2016) 149:186-203. doi: 10.1111/imm. 12626

37. Turtle L, Solomon T. Japanese Encephalitis - the Prospects for New Treatments. Nat Rev Neurol (2018) 14:298-313. doi: 10.1038/nrneurol.2018.30

38. Xing Z, Zuo Z, Hu D, Zheng X, Wang X, Yuan L, et al. Influenza Vaccine Combined With Moderate-Dose PD1 Blockade Reduces Amyloid-Beta Accumulation and Improves Cognition in APP/PS1 Mice. Brain Behav Immun (2021) 91:128-41. doi: 10.1016/j.bbi.2020.09.015 
39. Koyanagi N, Imai T, Shindo K, Sato A, Fujii W, Ichinohe T, et al. Herpes Simplex Virus-1 Evasion of CD8+ T Cell Accumulation Contributes to Viral Encephalitis. J Clin Invest (2017) 127:3784-95. doi: 10.1172/JCI92931

40. Wang Z, Li G, Dou S, Zhang Y, Liu Y, Zhang J, et al. Tim-3 Promotes Listeria Monocytogenes Immune Evasion by Suppressing Major Histocompatibility Complex Class I. J Infect Dis (2020) 221:830-40. doi: 10.1093/infdis/jiz512

41. Downs I, Vijayan S, Sidiq T, Kobayashi KS. Cita/Nlrc5: A Critical Transcriptional Regulator of MHC Class I Gene Expression. Biofactors (2016) 42:349-57. doi: 10.1002/biof.1285

42. Holloway G, Fleming FE, Coulson BS. MHC Class I Expression in Intestinal Cells is Reduced by Rotavirus Infection and Increased in Bystander Cells Lacking Rotavirus Antigen. Sci Rep (2018) 8:67. doi: 10.1038/s41598-01718464-x

43. Meissner TB, Li A, Biswas A, Lee KH, Liu YJ, Bayir E, et al. NLR Family Member NLRC5 is a Transcriptional Regulator of MHC Class I Genes. Proc Natl Acad Sci U S A (2010) 107:13794-9. doi: 10.1073/pnas. 1008684107

44. Callis J. The Ubiquitination Machinery of the Ubiquitin System. Arabidopsis Book (2014) 12:e0174. doi: 10.1199/tab.0174

45. Heaton SM, Borg NA, Dixit VM. Ubiquitin in the Activation and Attenuation of Innate Antiviral Immunity. J Exp Med (2016) 213:1-13. doi: 10.1084/ jem.20151531

46. Quinn LL, Williams LR, White C, Forrest C, Zuo J, Rowe M. The Missing Link in Epstein-Barr Virus Immune Evasion: The BDLF3 Gene Induces
Ubiquitination and Downregulation of Major Histocompatibility Complex Class I (Mhc-I) and MHC-II. J Virol (2016) 90:356-67. doi: 10.1128/ JVI.02183-15

47. Stamper CC, Zhang Y, Tobin JF, Erbe DV, Ikemizu S, Davis SJ, et al. Crystal Structure of the B7-1/CTLA-4 Complex That Inhibits Human Immune Responses. Nature (2001) 410:608-11. doi: 10.1038/35069118

48. Anderson AC. Tim-3: An Emerging Target in the Cancer Immunotherapy Landscape. Cancer Immunol Res (2014) 2:393-8. doi: 10.1158/2326-6066.CIR14-0039

49. Yang X, Jiang X, Chen G, Xiao Y, Geng S, Kang C, et al. T Cell Ig Mucin-3 Promotes Homeostasis of Sepsis by Negatively Regulating the TLR Response. J Immunol (2013) 190:2068-79. doi: 10.4049/jimmunol.1202661

Conflict of Interest: The authors declare that the research was conducted in the absence of any commercial or financial relationships that could be construed as a potential conflict of interest.

Copyright (C) 2021 Li, Tang, Hou, Wang, Gao, Dou, Mo, Hao, Gao, Li, Dong, Zhang, Shen, Wang and Han. This is an open-access article distributed under the terms of the Creative Commons Attribution License (CC BY). The use, distribution or reproduction in other forums is permitted, provided the original author(s) and the copyright owner(s) are credited and that the original publication in this journal is cited, in accordance with accepted academic practice. No use, distribution or reproduction is permitted which does not comply with these terms. 